\title{
Nonlocal effects on magnetism in the diluted magnetic semiconductor $\mathrm{Ga}_{1-x} \mathrm{Mn}_{x} \mathrm{As}$
}

\author{
Unjong Yu, ${ }^{1,2}$ Abdol-Madjid Nili, ${ }^{1}$ Karlis Mikelsons,,${ }^{1,3}$ Brian Moritz, ${ }^{4}$ Juana Moreno, ${ }^{1}$ and Mark Jarrell ${ }^{1}$ \\ ${ }^{1}$ Department of Physics and Astronomy $\&$ Center for Computation and Technology, \\ Louisiana State University, Baton Rouge, LA 70803, USA \\ ${ }^{2}$ Department of Applied Physics, Gwangju Institute of Science and Technology, Gwangju 500-712, Korea \\ ${ }^{3}$ Department of Physics, University of Cincinnati, Cincinnati, Ohio 45221, USA \\ 4 Stanford Institute for Materials and Energy Science, \\ SLAC National Accelerator Laboratory and Stanford University, Stanford, CA 94305, USA
}

(Dated: October 30, 2018)

\begin{abstract}
The magnetic properties of the diluted magnetic semiconductor $\mathrm{Ga}_{1-x} \mathrm{Mn}_{x}$ As are studied within the dynamical cluster approximation. We use the $\mathbf{k} \cdot \mathbf{p}$ Hamiltonian to describe the electronic structure of GaAs with spin-orbit coupling and strain effects. We show that nonlocal effects are essential for explaining the experimentally observed transition temperature and saturation magnetization. We also demonstrate that the cluster anisotropy is very strong and induces rotational frustration and a cube-edge direction magnetic anisotropy at low temperature. With this, we explain the temperature-driven spin reorientation in this system.
\end{abstract}

PACS numbers: 75.50.Pp, 75.30.Gw, 78.55.Cr

The discovery of high temperature ferromagnetism in diluted magnetic semiconductors (DMS) has stimulated a great deal of attention [1]. The interest in these materials is due to possible applications in spintronics [2] as the source of a spin polarized current or as the base material for a chip that can simultaneously store and process data.

In spite of extensive studies, our understanding of ferromagnetism in these systems is far from complete [3]. There are a few serious difficulties in the theoretical study of DMS: (i) The magnetic interaction between local magnetic moments and itinerant carrier spin, which is responsible for the high transition temperature $\left(T_{c}\right)$, is strong and outside the Ruderman-Kittel-Kasuya-Yosida (RKKY) regime. (ii) There exists strong disorder from the random distribution of magnetic ions. (iii) Nonlocal effects are expected to be crucial judging from the spatially oscillating and anisotropic magnetic interaction predicted in theory [4-7] and observed in experiments [8].

The mean-field study by Dietl et al. [9] captures the main features of DMS systems qualitatively and some even quantitatively. However, it ignores strong correlations, disorder effects, and spatial fluctuations, and fails to describe some DMS materials, such that subsequent studies have brought their approach into question [10, 11]. Studies [12 based on the dynamical mean-field theory (DMFT) [13] have made considerable improvements by including strong correlation and disorder effects. However, the local nature of the DMFT presents severe limitations when studying this system. The effects of short-range fluctuations and spatial correlations were shown to be important in the classical Heisenberg model [14]. In this letter, we show that nonlocal effects may be equally important for the itinerant carriers, which mediate the effective interaction between local moments in DMS.

The dynamical cluster approximation (DCA) [15] systematically incorporates nonlocal effects as the cluster size $\left(N_{c}\right)$ increases while retaining strong correlations.
When $N_{c}=1$, the DCA is equivalent to the DMFT, and exact results are approached as $N_{c} \rightarrow \infty$. Since all the possible disorder configurations are considered in a cluster, the DCA is also a better approximation for disorder average than the coherent potential approximation or DMFT by including multi-impurity scattering terms [16]. Thus, the DCA is an ideal method for studying DMS systems. In this Letter, we study the magnetic properties of the prototypical DMS system $\mathrm{Ga}_{1-x} \mathrm{Mn}_{x}$ As using the DCA and the $\mathbf{k} \cdot \mathbf{p}$ method, which describes the noninteracting band structure of pure GaAs. We show that nonlocal effects are very important for properly capturing the magnitude of $T_{c}$, the saturation magnetization, and the magnetic anisotropy of this material. In particular, we show that the strong cluster anisotropy is responsible for the magnetic anisotropy along the cube-edge direction and the spin reorientation at low temperature.

The model Hamiltonian we adopt is

$$
H=H_{\mathbf{k} \cdot \mathbf{p}}+J_{c} \sum_{I} \mathbf{S}\left(R_{I}\right) \cdot \mathbf{J}\left(R_{I}\right),
$$

where the first term describes the electronic structure of the host material (GaAs) in the $\mathbf{k} \cdot \mathbf{p}$ approximation and the second term introduces a magnetic interaction between the carrier spin $(\mathbf{J})$ and the local magnetic moment $(\mathbf{S})$ of $\mathrm{Mn}$ at position $R_{I}$. The large magnitude of the Mn magnetic moment $(S=5 / 2)$ allows us to treat it classically. This model is generally accepted to describe DMS [4, 15, 9, 12, 17, 18], since a mean-field treatment of the Hamiltonian [9, 17, 18] is able to explain many physical properties of the system. For the $\mathbf{k} \cdot \mathbf{p}$ Hamiltonian $\left(H_{\mathbf{k} \cdot \mathbf{p}}\right)$, we adopt a $4 \times 4$ Luttinger-Kohn model describing heavy and light hole bands with spin-orbit coupling, but ignoring the conduction and split-off bands. We use the Luttinger parameters $\gamma_{1}=6.98, \gamma_{2}=2.06$, and $\gamma_{3}=2.93$ [19]. Biaxial strain is included in $H_{\mathbf{k} \cdot \mathbf{p}}$ through the strain tensor $\varepsilon_{x x}=\varepsilon_{y y}=\varepsilon_{0}=\Delta a / a$ and $\varepsilon_{z z}=\left(-2 c_{12} / c_{11}\right) \varepsilon_{0}$ with the ratio of elastic stiffness 

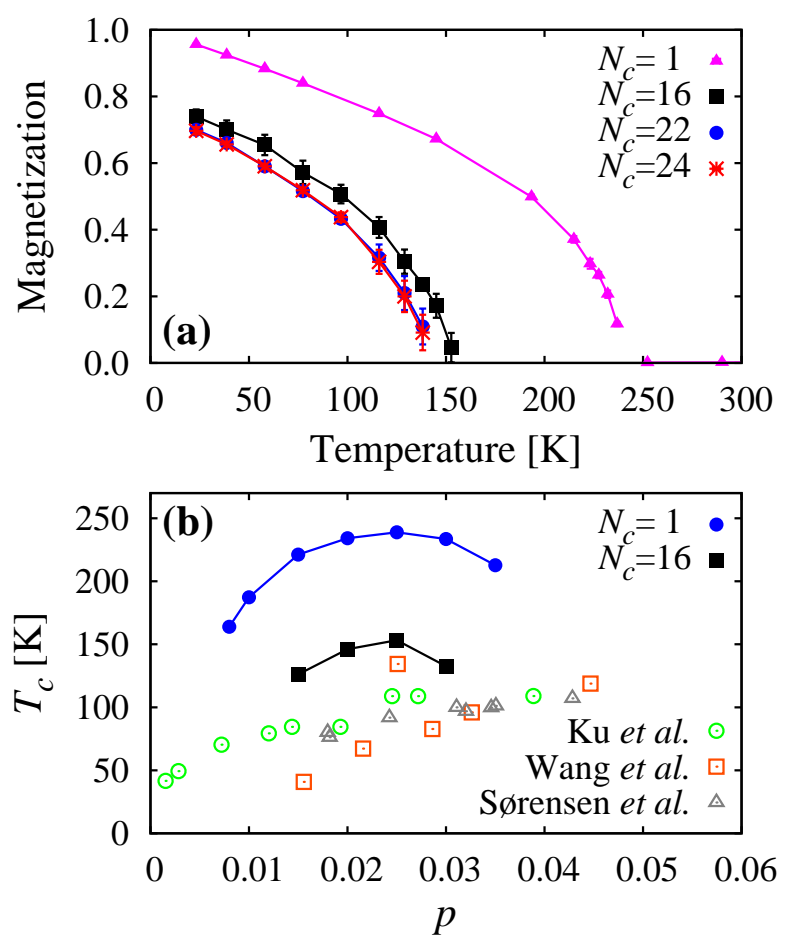

FIG. 1: (Color online) (a) Magnetization of $\mathrm{Ga}_{1-x} \mathrm{Mn}_{x} \mathrm{As}$ calculated by DMFT $\left(N_{c}=1\right)$ and DCA $\left(N_{c}=16, N_{c}=22\right.$, and $\left.N_{c}=24\right)$ with $\mathrm{Mn}$ doping $x=0.05$ and hole concentration $p=0.025$. No strain effect is considered. (b) Ferromagnetic transition temperature $\left(T_{c}\right)$ as a function of hole concentration $(p)$ with DMFT $\left(N_{c}=1\right)$ and DCA $\left(N_{c}=16\right)$ for $x=0.05$. Experimental results [26 28] are also shown. The $\mathrm{Mn}$ concentration in experiments is $x=0.085$ (Ku et al.), $x=0.017-0.09$ (Wang et al.), and $x=0.05$ (Sørensen et al.).

constants $c_{12} / c_{11}=0.46$. Parameter $a$ is the lattice constant of $\mathrm{Ga}_{1-x} \mathrm{Mn}_{x} \mathrm{As}$, and $\Delta a$ is the difference between the lattice constants of $\mathrm{Ga}_{1-x} \mathrm{Mn}_{x}$ As and the substrate. We use the hydrostatic deformation potential $a_{v}=1.16$ $\mathrm{eV}$ and the shear deformation potential $b=-2.0 \mathrm{eV}[19]$.

In addition to the parameters of the $\mathbf{k} \cdot \mathbf{p}$ Hamiltonian, we must determine the value of the exchange coupling $J_{c}$. It can be obtained from photoemission [20], infrared [21, 22] , and resonant tunneling 23] spectroscopy and magneto-transport experiments [24], which give $J_{c}=0.6$ $1.5 \mathrm{eV}$. We adopt $J_{c}=1 \mathrm{eV}$ throughout this Letter.

Figure1(a) shows the magnetization per $\mathrm{Mn}$ ion of the $\mathrm{Ga}_{1-x} \mathrm{Mn}_{x}$ As system as a function of temperature with $\operatorname{DMFT}\left(N_{c}=1\right)$ and DCA $\left(N_{c}=16, N_{c}=22\right.$, and $\left.N_{c}=24\right)$. We chose three fcc clusters that are perfect according to Betts et al. 25]. The difference between DCA and DMFT stems from nonlocal effects, not captured in DMFT. The $T_{c}$ with DCA is far lower than that obtained with DMFT, approaching the regime of experimental values 26 28] [see Fig. 1(b)]. Another important point is the reduction of the saturation magnetization at low temperature, consistent with experiments [28 30$]$. This behavior is a product of the rotational frustration [4], to be discussed in detail later. This effect also re-

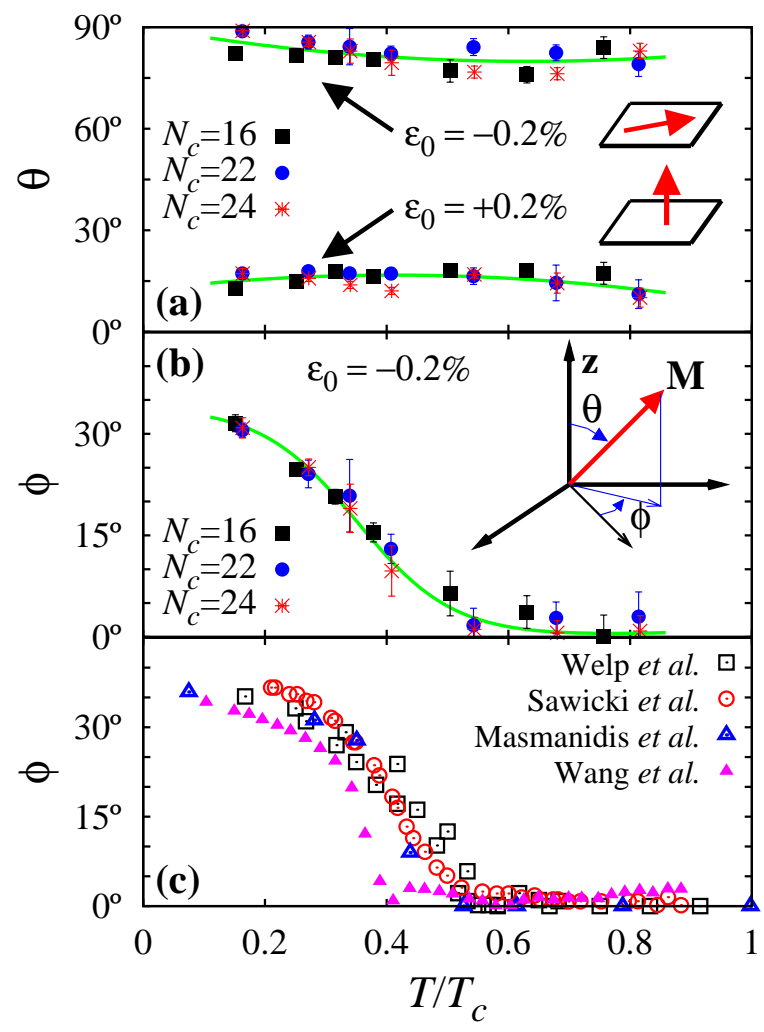

FIG. 2: (Color online) (a) Polar angle $(\theta)$ of the magnetization as a function of the normalized temperature $\left(T / T_{c}\right)$ for two strain values. Compressive $\left(\varepsilon_{0}=-0.2 \%\right)$ and tensile $\left(\varepsilon_{0}=+0.2 \%\right)$ strain induce in-plane and perpendicular magnetic anisotropy, respectively. (b) Azimuthal angle $(\phi)$ of the magnetization with respect to the [110] direction with compressive strain. Experimental results 31 34 are provided in (c) to compare with (b).

duces $T_{c}$. The dependence of $T_{c}$ on hole concentration $(p)$ is shown in Fig. 1(b). $T_{c}$ attains a maximum value when hole concentration is half of Mn concentration, consistent with previous DMFT studies [12].

Next, we studied the magnetic anisotropy of $\mathrm{Ga}_{1-x} \mathrm{Mn}_{x}$ As. The magnetic anisotropy of this system depends on strain, hole concentration, and temperature in a complicated manner, but generally it has in-plane anisotropy with compressive strain and perpendicular-toplane anisotropy with tensile strain [1]. With compressive strain, the magnetization changes direction within plane from [110] or [150] at high temperature to [100] or [010] at low temperature 31 34] [see Fig. 2(c)]. As is shown in Fig. 2(a) and 2(b), the DCA reproduces experimental results on the dependence of magnetic anisotropy on strain and temperature remarkably well.

While the strain dependence of the magnetic anisotropy was explained within the mean-field theory [17, 18], the spin reorientation within the plane has not been explained yet. In the absence of strain, $\mathrm{Ga}_{1-x} \mathrm{Mn}_{x}$ As has diagonal magnetic anisotropy within the mean-field theory because the heavy holes, which dominate at low carrier density, have larger density along 


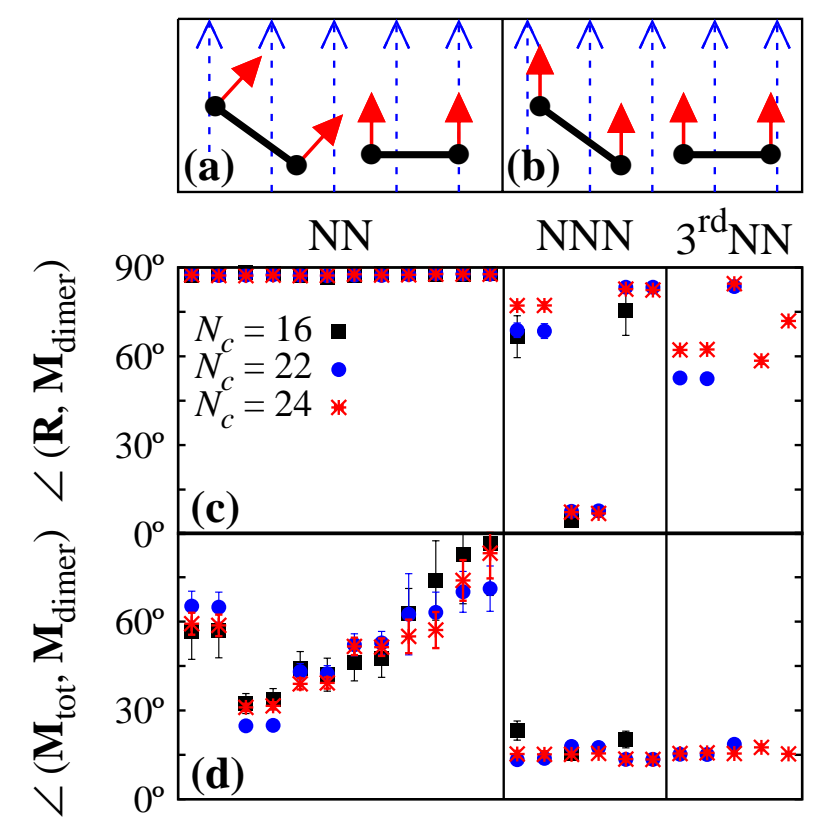

FIG. 3: (Color online) (a) DCA results at low temperature show that the dimer magnetization $\left(\mathbf{M}_{\text {dimer }}\right)$ is prefered to be perpendicular to the vector connecting the two $\mathrm{Mn}$ ions (R) for nearest-neighbor (NN) Mn-dimers. (b) For larger dimers, $\mathbf{M}_{\text {dimer }}$ aligns with the total magnetization $\left(\mathbf{M}_{\text {tot }}\right)$ irrespective of $\mathbf{R}$. The dotted and solid arrows represent $\mathbf{M}_{\text {tot }}$ and $\mathbf{M}_{\text {dimer }}$, respectively. (c) and (d) Angle between $\mathbf{M}_{\text {dimer }}$ and $\mathbf{R}$ and between $\mathbf{M}_{\text {dimer }}$ and $\mathbf{M}_{\text {tot }}$, respectively, at $T=23.2 \mathrm{~K}$, $x=0.05, p=0.025$, and $\varepsilon_{0}=-0.2 \%$. The left panel is for the $12 \mathrm{NN}$ dimers [e.g., when the two $\mathrm{Mn}$ ions are at $(0,0,0)$ and $(a / 2, a / 2,0)]$, the middle panel is for the 6 next-nearestneighbor $(\mathrm{NNN})$ dimers [e.g., when the two $\mathrm{Mn}$ ions are at $(0,0,0)$ and $(a, 0,0)]$, and the right panel is for the thirdnearest-neighbor $\left(3^{\mathrm{rd}} \mathrm{NN}\right)$ dimers [e.g., when the two $\mathrm{Mn}$ ions are at $(0,0,0)$ and $(a, a / 2, a / 2)]$.

the diagonal direction in $\mathbf{k}$-space [17]. With compressive strain, it has the anisotropy in [110] or [110]. This explains the magnetic anisotropy at high temperature.

The [100] or [010] anisotropy at low temperature is due to the cluster anisotropy originating from the anisotropic interaction between neighboring Mn ions. Because Mn ions are distributed randomly throughout the system, the number of $\mathrm{Mn}$ ions within a cluster varies between zero and $N_{c}$. We call a cluster a monomer (dimer) when it includes one $\mathrm{Mn}$ ion (two Mn ions). All possible distributions of $\mathrm{Mn}$ ions are considered effectively in this calculation, but at low doping, the magnetic properties are dominated by Mn-monomers and Mn-dimers. Since there is no cluster anisotropy in monomers, we investigate magnetization of Mn-dimers in detail. Because of translational symmetry, we need to consider only $N_{c}-1$ dimers. The two Mn ions are nearest-neighbors in 12 dimers, next-nearest-neighbors in 6 dimers, and thirdnearest-neighbors in $N_{c}-19$ dimers. Figure 3 shows the magnetization direction of each dimer obtained by the Monte-Carlo method at low temperature. The magnetization of the nearest-neighbor Mn-dimer is always
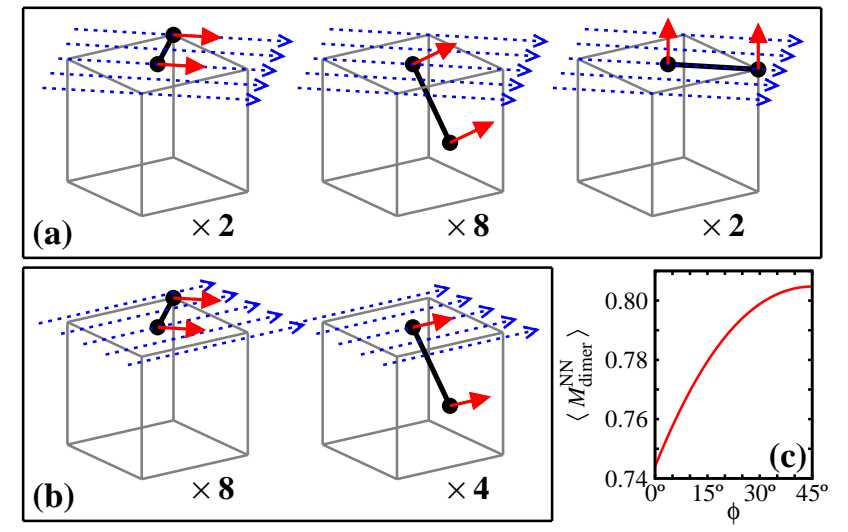

FIG. 4: (Color online) (a) Magnetic configurations of the 12 nearest-neighbor (NN) Mn-dimers $\left(\mathbf{M}_{\text {dimer }}^{\mathrm{NN}}\right)$ that maximize the total magnetization $\left(\mathbf{M}_{\text {tot }}\right)$ when $\phi=0^{\circ}$ (i.e. $\mathbf{M}_{\text {tot }}$ is along [110] or [1]10]). The dotted and solid arrows represent $\mathbf{M}_{\text {tot }}$ and $\mathbf{M}_{\text {dimer }}^{\mathrm{NN}}$, respectively. The numbers below each diagram indicate the degeneracy of the Mn-dimer configurations. The dimer configuration with $\mathbf{M}_{\mathrm{dimer}}^{\mathrm{NN}}$ perpendicular to the vector connecting the two $\mathrm{Mn}$ ions $(\mathbf{R})$ is energetically favored. (b) Same as (a) but when $\phi=45^{\circ}$ (i.e. $\mathbf{M}_{\text {tot }}$ is along [100] or [010]). (c) Maximum value of the average $\mathbf{M}_{\text {dimer }}^{\text {NN }}$ vs. $\phi$.

perpendicular to the vector connecting the two Mn ions. This cluster anisotropy prevents the magnetic moment of some dimers from aligning parallel to the total magnetization and leads to the rotational frustration [4, 5]. When the two Mn ions are farther apart, this anisotropy is very weak and magnetization of the dimer aligns parallel to the total magnetization.

The cluster anisotropy can also explain the enhancement of $T_{c}$ up to $260 \mathrm{~K}$ in the quasi-two-dimensional $\delta$-doped systems [35]. When the Mn ions are within one plane, all the nearest-neighbor Mn-dimers can point in the same direction (perpendicular-to-plane direction) without rotational frustration. This may induce a larger saturation magnetization and higher $T_{c}$ in these systems.

Notably, due to this cluster anisotropy, the maximum total magnetization depends on the magnetization direction, and this dependence introduces another type of magnetic anisotropy. When we assume the magnetic moment is perpendicular to the vector connecting the two $\mathrm{Mn}$ ions and in-plane magnetization, the maximum value of the average magnetization of the 12 nearest-neighbor dimers is calculated to be

$$
\begin{aligned}
\left\langle M_{\mathrm{dimer}}^{\mathrm{NN}}\right\rangle=\frac{1}{6} & {[\cos \phi+\sin \phi+\sqrt{3+\sin (2 \phi)}} \\
& +\sqrt{3-\sin (2 \phi)}]
\end{aligned}
$$

for the fcc lattice. This becomes maximal when the magnetization is along [100] or [010], as shown in Fig. 4(c). Since larger magnetization leads to lower magnetic energy, it introduces magnetic anisotropy along [100] or [010]. We note that this effect becomes unimportant at high temperature, where the total magnetization is 
small. Thus, the spin reorientation from [110] or [110] at high temperature to [100] or [010] at low temperature is captured within our calculation. This behavior arises from the multi-impurity scattering and cannot be obtained within the mean-field theory or the DMFT.

In summary, we investigated the magnetic properties of the prototypical DMS system $\mathrm{Ga}_{1-x} \mathrm{Mn}_{x}$ As by DCA together with the $\mathbf{k} \cdot \mathbf{p}$ method. We showed that nonlocal effects, not included in the mean-field theory or the DMFT but included in the DCA for $N_{c}>1$, are very important to quantitatively explain the $T_{c}$, the saturation magnetization, and the magnetic anisotropy of this material. We find that the edge-direction anisotropy at low temperature is due to the cluster anisotropy, and this allows us to reproduce the spin reorientation in this system remarkably well.

We acknowledge helpful discussions with $\mathrm{Z}$. Xu. This work was supported by the National Science Foundation through OISE-0730290, DMR-0548011, and DMR0706379. BM acknowledges support from the U.S. Department of Energy, Office of Basic Energy Sciences, under contract DE-AC02-76SF00515. Portions of this research were conducted with high performance computational resources provided by the Louisiana Optical Network Initiative (http://www.loni.org).

[1] H. Ohno, Science 281, 951 (1998).

[2] Spintronics, edited by T. Dietl, D. D. Awschalom, M. Kaminska, and H. Ohno (Academic Press, New York, 2008).

[3] For a review, see T. Jungwirth, J. Sinova, J. Mašek, J. Kuěra, A. H. MacDonald, Rev. Mod. Phys. 78, 809 (2006).

[4] G. Zaránd and B. Jankó, Phys. Rev. Lett. 89, 047201 (2002).

[5] J. Moreno, R. S. Fishman, and M. Jarrell, Phys. Rev. Lett. 96, 237204 (2006).

[6] P. Mahadevan, A. Zunger, and D. D. Sarma, Phys. Rev. Lett. 93, 177201 (2004).

[7] J. Kudrnovský, I. Turek, V. Drchal, F. Máca, P. Weinberger, and P. Bruno, Phys. Rev. B 69, 115208 (2004).

[8] D. Kitchen, A. Richardella, J.-M. Tang, M. E. Flatté, and A. Yazdani, Nature 442, 436 (2006).

[9] T. Dietl, H. Ohno, F. Matsukura, J. Cibert, D. Ferrand, Science 287, 1019 (2000).

[10] D. J. Priour, Jr., E. H. Hwang, and S. Das Sarma, Phys. Rev. Lett. 92, 117201 (2004).

[11] R. Bouzerar, G. Bouzerar, and T. Ziman, Phys. Rev. B 73, 024411 (2006).

[12] A. Chattopadhyay, S. Das Sarma, and A. J. Millis, Phys. Rev. Lett. 87, 227202 (2001); L. Craco, M. S. Laad, and E. Müller-Hartmann, Phys. Rev. B 68, 233310 (2003); K. Aryanpour, J. Moreno, M. Jarrell, and R. S. Fishman, Phys. Rev. B 72, 045343 (2005); F. Popescu, Y. Yildirim, G. Alvarez, A. Moreo, and E. Dagotto, Phys. Rev. B 73, 075206 (2006).

[13] W. Metzner and D. Vollhardt, Phys. Rev. Lett. 62, 324 (1989); A. Georges, G. Kotliar, W. Krauth, and
M. Rozenberg, Rev. Mod. Phys. 68, 13 (1996).

[14] R. N. Bhatt and X. Wan, Int. J. Mod. Phys. C 10, 1459 (1999); L. Bergqvist, O. Eriksson, J. Kudrnovský, V. Drchal, P. Korzhavyi, and I. Turek, Phys. Rev. Lett. 93, 137202 (2004); K. Sato, W. Schweika, P. H. Dederichs, H. Katayama-Yoshida, Phys. Rev. B 70, 201202(R) (2004).

[15] For a review, see T. Maier, M. Jarrell, T. Pruschke, M. H. Hettler, Rev. Mod. Phys. 77, 1027 (2005).

[16] M. Jarrell and H. R. Krishnamurthy, Phys. Rev. B 63, 125102 (2001); B. Moritz, K. Mikelsons, J. Moreno, R. Fishman, and M. Jarrell (unpublished).

[17] M. Abolfath, T. Jungwirth, J. Brum, and A. H. MacDonald, Phys. Rev. B 63, 054418 (2001).

[18] T. Dietl, H. Ohno, and F. Matsukura, Phys. Rev. B 63, 195205 (2001).

[19] J. Piprek, Semiconductor Optoelectronic Devices (Academic Press, San Diego, 2003).

[20] J. Okabayashi, A. Kimura, O. Rader, T. Mizokawa, A. Fujimori, T. Hayashi, and M. Tanaka, Phys. Rev. B 58, R4211 (1998).

[21] M. Linnarsson, E. Janzén, B. Monemar, M. Kleverman, and A. Thilderkvist, Phys. Rev. B 55, 6938 (1997); A. K. Bhattacharjee and C. B. à la Guillaume, Solid State Commun. 113, 17 (1999).

[22] E. J. Singley, K. S. Burch, R. Kawakami, J. Stephens, D. D. Awschalom, D. N. Basov, Phys. Rev. B 68, 165204 (2003).

[23] H. Ohno, N. Akiba, F. Matsukura, A. Shen, K. Ohtani, and Y. Ohno, Appl. Phys. Lett. 73, 363 (1998).

[24] T. Omiya, F. Matsukura, T. Dietl, Y. Ohno, T. Sakon, M. Motokawa, H. Ohno et al., Physica E 7, 976 (2000).

[25] D. D. Betts, S. Masui, N. Vats, and G. E. Stewart, Can. J. Phys. 74, 54 (1996).

[26] B. S. Sørensen, P. E. Lindelof, J. Sadowski, R. Mathieu, and P. Svedlindh, Appl. Phys. Lett. 82, 2287 (2003).

[27] K. C. Ku, S. J. Potashnik, R. F. Wang, S. H. Chun, P. Schiffer, N. Samarth, M. J. Seong, A. Mascarenhas, E. Johnston-Halperin, R. C. Myers, A. C. Gossard, and D. D. Awschalom, Appl. Phys. Lett. 82, 2302 (2003).

[28] K. Y. Wang, K. W. Edmonds, R. P. Campion, B. L. Gallagher, N. R. S. Farley, C. T. Foxon, M. Sawicki, P. Boguslawski, and T. Dietl, J. Appl. Phys. 95, 6512 (2004).

[29] A. Van Esch, L. Van Bockstal, J. De Boeck, G. Verbanck, A. S. van Steenbergen, P. J. Wellmann, B. Grietens, R. Bogaerts, F. Herlach, and G. Borghs, Phys. Rev. B 56, 13103 (1997).

[30] S. J. Potashnik, K. C. Ku, R. Mahendiran, S. H. Chun, R. F. Wang, N. Samarth, and P. Schiffer, Phys. Rev. B 66, 012408 (2002).

[31] M. Sawicki, F. Matsukura, A. Idziaszek, T. Dietl, G. M. Schott, C. Ruester, C. Gould, G. Karczewski, G. Schmidt, and L. W. Molenkamp, Phys. Rev. B 70, 245325 (2004).

[32] K.-Y. Wang, M. Sawicki, K. W. Edmonds, R. P. Campion, S. Maat, C. T. Foxon, B. L. Gallagher, and T. Dietl, Phys. Rev. Lett. 95, 217204 (2005).

[33] U. Welp, V. K. Vlasko-Vlasov, X. Liu, J. K. Furdyna, and T. Wojtowicz, Phys. Rev. Lett. 90, 167206 (2003).

[34] S. C. Masmanidis, H. X. Tang, E. B. Myers, M. Li, K. De Greve, G. Vermeulen, W. Van Roy, and M. L. Roukes, Phys. Rev. Lett. 95, 187206 (2005).

[35] A. M. Nazmul, T. Amemiya, Y. Shuto, S. Sugahara, and M. Tanaka, Phys. Rev. Lett. 95, 017201 (2005). 